\title{
Justification of the Averaging Method for the Boundary Value Problems on a Finite or Semi-Infinite Interval
}

\author{
V. B. Levenshtam, P. E. Shubin
}

\begin{abstract}
We justify the averaging technique for the boundary value problems for the normal ODE systems involving large parameters on a finite or semi-infinite interval.

Keywords: Normal ODE system, boundary value problems, high frequency, averaging method.
\end{abstract}

\section{Introduction}

The prior advance towards the justification of the averaging for the boundary value problems for the normal ODE systems involving large parameters on a finite or semi-infinite interval had been achieved in [1]. However, the result of [1] involves substantial restriction. Namely, the Jacoby matrix of the nonlinear terms of the averaged system is required to vanish on the solution of it. In this paper, this restriction is removed.

\section{Auxiliary information.}

This section consist of three subsections. In subsection 2.1 we introduce notations used throughout the paper; in subsections 2.2 and 2.3 we present auxiliary information.

Let $J$ denote a fixed segment $[a, b]$ or semiaxis $[0, \infty)$ and $n$ - fixed positive integer. Let $M(J)$ be set of real square matrices of order $n$ whose elements are continuous on $J$.

We recall the definitions of the following Banach spaces: $C(J)$ is space of continuous vector functions $u: J \rightarrow \mathbb{R}^{n}$ with sup-norm; $C^{\mu}(J), \mu \in(0,1)$ is space of vector functions $u(t) \in C(J)$, satisfying the condition Holder with exponent $\mu$, i. e.

$$
\|u\|_{C^{\mu}(J)}=\|u\|_{C(J)}+\sup _{\substack{t_{1}, t_{2} \in J \\ t_{1}<t_{2}}} \frac{\left|u\left(t_{2}\right)-u\left(t_{1}\right)\right|}{\left(t_{2}-t_{1}\right)^{\mu}}<\infty .
$$

Manuscript received January 21, 2015; accepted May 10, 2015.

V. B. Levenshtam, P. E. Shubin are with the the Department of Algebra and Discrete Mathematics, Southern Federal University, 344090, Rostov-on-Don, Milchakov 8A, Russia 
This subsection contains auxiliary information regarding the problem on the segment $J=[a, b]$.

We consider the boundary value problem

$$
\left\{\begin{aligned}
\frac{d}{d t} y(t)=A(t) y(t) & +f(t), \quad t \in J \\
L y(a) & =l \\
R y(b) & =r
\end{aligned}\right.
$$

Here $A(t) \in M(J), f(t) \in C(J), L$ and $R$ are real matrices having $n \in \mathbb{N}$ columns and $k_{L} \in \mathbb{N}$, $k_{R} \in \mathbb{N}$ rows respectively, $l$ and $r$ are $k_{L}$ and $k_{R}$ - dimensional real vector-columns respectively, $y(t)$ is unknown $n$ - dimensional vector-function.

Proposition 1 The necessary and sufficient conditions for existence and uniqueness of the solution of problem (1) are as follows:

1) $k_{L}+k_{R}=n$,

2) $\operatorname{det}\left[\begin{array}{c}L \\ R \Omega(a, b)\end{array}\right] \neq 0$.

Here $\Omega(a, b)$ denotes matriciant (see.[2]) of homogeneous ( $f=0$ ) boundary value problem (1).

Definition 1 Matrix-functions $G(t, s) \in[a, b] \times[a, b], G_{L}(t)$ and $G_{R}(t) \in[a, b]$ with $n$ rows and $n$ columns, $n$ rows and $k_{L}$ columns, $n$ rows and $k_{R}$ columns respectively are said to be Green's matrices of problem (1) if they satisfy the following conditions:

$$
\begin{aligned}
& \left\{\begin{array}{l}
G(s+0, s)-G(s-0, s)=I_{n}, \\
\frac{d}{d t} G(t, s)=A(t) G(t, s) t \neq s, \\
L G_{L}(a, s)=0, \quad R G_{R}(b, s)=0 ;
\end{array}\right. \\
& \left\{\begin{array}{l}
\frac{d}{d t} G_{L}(t)=A(t) G_{L}(t) \\
L G_{L}(a)=I_{k_{L}}, \quad R G_{L}(b)=0
\end{array}\right. \\
& \left\{\begin{aligned}
\frac{d}{d t} G_{R}(t) & =A(t) G_{R}(t), \\
L G_{R}(a) & =0, \quad R G_{R}(b)=I_{k_{R}}
\end{aligned}\right.
\end{aligned}
$$


In the case of a constant matrix $A(t) \equiv A$ the construction of matrices $G, G_{L}, G_{R}$ and the proof of proposition 1 has been proved in [3, ch. I, §11]. In the case of variable matrix $A(t)$ - the similar reasonings works.

Nevertheless, we describe the derivation of the Green's matrices for the sake of completeness of exposition in subsection 4.1.

This subsection contains auxiliary information regarding the problem on the semiaxis $J=[0, \infty)$.

We consider a homogeneous ordinary differential equation on the $J$

$$
\frac{d}{d t} x(t)=A(t) x(t), \quad A(t) \in M(J)
$$

We assume, that $A(t)$ is integrally bounded, i.e. there exist $M>0$ such that for any $t \in J$ hold $\int_{t}^{t+1}|A(\tau)| d \tau \leq M$

Definition 2 (see, example, [4, §3]) The solutions of equation (2) admit an exponential dichotomy on the semiaxis, if $\mathbb{R}^{n}=B_{1} \oplus B_{2}$ and there exist $v>0$ such that subspaces $B_{1}$, $B_{2}$ and $v$ obey the following conditions:

a) solutions $x_{1}(t)=U(t) x_{1}^{0}$ of the equation 2, leaving at time $t=0$ from the subspace $B_{1}$, satisfy the estimate

$$
\left|x_{1}(t)\right| \leq N_{1} \exp (-v(t-s))\left|x_{1}(s)\right| \quad(t \geq s ; t, s \in J)
$$

b) solutions $x_{2}(t)=U(t) x_{2}^{0}$ of the equation (2), leaving at time $t=0$ from the subspace $B_{2}$, satisfy the estimate

$$
\left|x_{2}(t)\right| \leq N_{2} \exp \left(-v_{2}(s-t)\right)\left|x_{2}(s)\right| \quad(s \geq t ; t, s \in J)
$$

In this paper we employ the criterion of exponential dichotomy on the semiaxis given in [4, ch.IV, corallary 3.1].

Proposition $2 O D E(2)$ with an integrally bounded matrix function $A(t)$ possesses the exponential dichotomy on the semiaxis if and only if for every $f(t) \in C(J)$ there exists at least one bounded solution of the inhomogeneous equation.

\section{Main results}

This section consists of two subsections. In the former subsection we treat the case of $J=[a, b]$ and in the latter subsection we treat the case of $J=[0, \infty)$. 
We consider the boundary value problem on a segment $t \in J=[a, b]$ for the normal system of ordinary differential equations

$$
\left\{\begin{array}{c}
\frac{d x}{d t}=f(x, t, \omega t) \quad \omega \gg 1 \\
L x(a)=l \\
R x(b)=r .
\end{array}\right.
$$

Here $L, R, l$ and $r$ are matrices and vectors-columns, that have been defined in subsection 2.2, $x(t)$ is unknown $n$ - dimensional vector-function.

We make the following assumption about problem (3).

1. Vector function $f(x, t, \tau)$ with values in $\mathbb{R}^{n}$ is continuous on the set $\Sigma \equiv S \times J \times[0 ; \infty)$ where $S$ is domain in $\mathbb{R}^{n}$ and continuously differentiable in $x \in \Sigma$.

2. Vector function $f(x, t, \tau)$ and Jacoby matrix $f_{x}^{\prime}(x, t, \tau)$ are bounded on $\Sigma$, i. e. for any $(x, t, \tau) \in \Sigma \quad|f(x, t, \tau)| \leq C_{1}$ and $\left|f_{x}^{\prime}(x, t, \tau)\right| \leq C_{1}$ where $C_{1}=$ const

3. Vector function $f(x, t, \tau)$ and Jacoby matrix $f_{x}^{\prime}(x, t, \tau)$ satisfy uniform Holder condition in $t$, that is, for any $\left(x, t_{1}, \tau\right),\left(x, t_{2}, \tau\right) \in \Sigma\left|f\left(x, t_{2}, \tau\right)-f\left(x, t_{1}, \tau\right)\right| \leq C_{2}\left|t_{2}-t_{1}\right|^{\gamma}$ and $\left|f_{x}^{\prime}\left(x, t_{2}, \tau\right)-f_{x}^{\prime}\left(x, t_{1}, \tau\right)\right| \leq C_{2}\left|t_{2}-t_{1}\right|^{\gamma}$ where $C_{2}=$ const,$\gamma \in(0 ; 1)$.

4. Jacoby matrix $f_{x}^{\prime}(x, t, \tau)$ satisfies uniform Lipschitz condition in $(x, t, \tau) \in \Sigma$ in $x$, i. e. for any $\left(x_{1}, t, \tau\right),\left(x_{2}, t, \tau\right) \in \Sigma\left|f_{x}^{\prime}\left(x_{2}, t, \tau\right)-f_{x}^{\prime}\left(x_{1}, t, \tau\right)\right| \leq C_{3}\left|x_{2}-x_{1}\right|$ where $C_{3}=$ const .

5. There exist limits

$$
F(x, t) \equiv \lim _{T \rightarrow \infty} \frac{1}{T} \int_{0}^{T} f(x, t, \tau) d \tau, \quad F_{x}^{\prime}(x, t) \equiv \lim _{T \rightarrow \infty} \frac{1}{T} \int_{0}^{T} f_{x}^{\prime}(x, t, \tau) d \tau
$$

and the limits are attained uniformly in $(x, t)$.

Together with perturbed problem (3) consider the averaged problem

$$
\left\{\begin{array}{c}
\frac{d y}{d t}=F(y, t) \\
L y(a)=l \\
R y(b)=r .
\end{array}\right.
$$

6. Assume that problem (4) has a solution $\stackrel{\circ}{y}(t)$. 
7. Let $k_{L}+k_{R}=n$ and $\operatorname{det}\left[\begin{array}{c}L \\ R \Omega(a, b)\end{array}\right] \neq 0$.

Theorema 1 Let the conditions $1-7$ be satisfied. Then there exists $\omega_{0}>0$ such that for every $\omega>\omega_{0}$ there exists a unique real solution $x_{\omega}$ of problem (3), which belongs to a neighborhood of $y$ in some $C^{\mu}([a, b])$ and

$$
\lim _{\omega \rightarrow \infty}\left\|x_{\omega}-\stackrel{\circ}{y}\right\|_{C^{\mu}([a ; b])}=0 .
$$

Let us consider the problem on the semiaxis $t \in J=[0, \infty)$

$$
\left\{\begin{array}{c}
\frac{d x}{d t}=f(x, t, \omega t) \quad \omega \gg 1 \\
M x(0)=\varphi
\end{array}\right.
$$

Here $M$ is matrix of boundary condition whose rows are linearly independent, $\varphi$ is a vector whose dimension coincides with the number of rows of matrix $M, x(t)$ is unknown $n$ dimensional vector-function.

We make the following assumption on the problem (5).

1. Vector function $f(x, t, \tau)$ with values in $\mathbb{R}^{n}$ is continuous on the set $\Sigma \equiv S \times J \times J$ where $S$ is domain in $\mathbb{R}^{n}$ and continuously differentiable in $x \in \Sigma$.

2. Vector function $f(x, t, \tau)$ and Jacoby matrix $f_{x}^{\prime}(x, t, \tau)$ are bounded on $\Sigma$, i. e. for any $(x, t, \tau) \in \Sigma \quad|f(x, t, \tau)| \leq C_{1}$ and $\left|f_{x}^{\prime}(x, t, \tau)\right| \leq C_{1}$ where $C_{1}=$ const.

3. Vector function $f(x, t, \tau)$ and Jacoby matrix $f_{x}^{\prime}(x, t, \tau)$ satisfy uniform Holder condition in $t$, that is, for any $\left(x, t_{1}, \tau\right),\left(x, t_{2}, \tau\right) \in \Sigma\left|f\left(x, t_{2}, \tau\right)-f\left(x, t_{1}, \tau\right)\right| \leq C_{2}\left|t_{2}-t_{1}\right|^{\gamma}$ and $\left|f_{x}^{\prime}\left(x, t_{2}, \tau\right)-f_{x}^{\prime}\left(x, t_{1}, \tau\right)\right| \leq C_{2}\left|t_{2}-t_{1}\right|^{\gamma}$ where $C_{2}=$ const,$\gamma \in(0 ; 1)$.

4. Jacoby matrix $f_{x}^{\prime}(x, t, \tau)$ satisfies uniform Lipschitz condition in $(x, t, \tau) \in \Sigma$ in $x$, i. e. for any $\left(x_{1}, t, \tau\right),\left(x_{2}, t, \tau\right) \in \Sigma\left|f_{x}^{\prime}\left(x_{2}, t, \tau\right)-f_{x}^{\prime}\left(x_{1}, t, \tau\right)\right| \leq C_{3}\left|x_{2}-x_{1}\right|$ where $C_{3}=$ const.

5. There exist limits

$$
F(x, t) \equiv \lim _{T \rightarrow \infty} \frac{1}{T} \int_{0}^{T} f(x, t, \tau) d \tau, \quad F_{x}^{\prime}(x, t) \equiv \lim _{T \rightarrow \infty} \frac{1}{T} \int_{0}^{T} f_{x}^{\prime}(x, t, \tau) d \tau
$$

and the limits are attained uniformly in $(x, t)$.

Along with perturbed problem (5) consider the averaged problem

$$
\left\{\begin{array}{l}
\frac{d y}{d t}=F(y, t) \\
M y(0)=\varphi
\end{array}\right.
$$


6. Problem (6) has a solution $\stackrel{\circ}{y}(t)$.

We also assume the following.

7. Norm of the matrix $A(t) \equiv F_{z}^{\prime}(\stackrel{\circ}{y}(t), t)$ is integrally bounded on the semiaxis, and for every continuous bounded vector function $g(t)$ there exists at least one bounded solution of the inhomogeneous equation $\frac{d u}{d t}=A(t) u+g(t)$ on the semiaxis $t \geq 0$.

Let $B_{1}, B_{2}$ be the subspaces (see, Definition 4.1) arising from exponential dichotomy of this homogeneous ( $g=0)$ equation on a semiaxis.

We denote the basis in the subspace $B_{1}$ as $\left\{e_{k}\right\}$. Let $S$ be the matrix consisting of vector-columns $e_{k}$.

The final condition is:

8. Matrix $M S$ is invertible.

Theorema 2 Let the conditions $1-8$ be satisfied. Then there exists $\omega_{0}>0$ such that for every $\omega>\omega_{0}$ there exists a unique real bounded solution $x_{\omega}$ of problem (5), which belongs to a neighborhood of $y$ in some $C^{\mu}([0, \infty))$ and

$$
\lim _{\omega \rightarrow \infty}\left\|x_{\omega}-\stackrel{\circ}{y}\right\|_{C^{\mu}([0 ; \infty))}=0 .
$$

\section{Method of proof}

Method of proof of theorems 1 and 2 includes three steps. First we reformulate the problems as an integral equation. Second, we associate the integral equation with an operator equations in appropriate spaces. Third we apply the abstract implicit function theorem. Earlier such scheme was used in [5], where the method of averaging is justified for abstract parabolic equations in the case of the Cauchy problem on a finite time interval and as well as for the problem of periodic solutions on the whole axis of time. In this paper we only describe the reduction of problems (3) and (5) to the integral equation (see 4.1 and 4.2). For the sake of briefness we omit the applying of the implicit function theorem since it is quite cumbersome and the very close technique had been exposed in [5] (see too [6]).

We need to build the Green matrix of the boundary value problem on a segment in order to get the integral equation. Such reduction is known for in the case of a constant matrix $A(t) \equiv A$ [3, see p.112]. In the case of variable matrix $A(t)$ the similar idea still works. Nevertheless, we describe the derivation of the Green's matrices for the sake of completeness of exposition. 
Lemma 1 ([3, see p.112]). If $k_{L}+k_{R}=n$ and det $\left[\begin{array}{c}L \\ R \Omega(a, b)\end{array}\right] \neq 0$, then for every $a<s<b$ and any vectors $l, r$ and $f$ having dimensions $k_{L}, k_{R}$ and $n$ respectively there exists vector function $x(t, s)$ which is continuously differentiable in $t$ on both interval $a \leq t<s$ and on interval $s<t \leq b$ and satisfies the homogeneous differential equation (2) on these intervals. This vector function has a discontinuity at point $t=s ;$ moreover,

$$
x(s+0, s)-x(s-0, s)=f .
$$

In addition, $x(t, s)$ satisfy boundary condition $L x(a, s)=l$ at point $t=a$ and boundary condition $R x(b, s)=r$ at point $t=b$.

For $s \in(a, b)$ and fixed vector $f \in \mathbb{R}^{n}$ we define

$$
x_{0}(t, s)=\left\{\begin{array}{l}
0, \quad a \leq t<s \\
\Omega(s, t) f, \quad s<t \leq b .
\end{array}\right.
$$

Obviously, for $t \neq s x_{0}(t, s)$ is continuously differentiable and

$$
\frac{d}{d t} x_{0}(t, s)=A(t) x_{0}(t, s) \quad t \neq s
$$

and $x_{0}(s+0, s)-x_{0}(s-0, s)=f($ see [2]).

Vector-function $x(t, s)=\Omega(a, t) m+x_{0}(t, s)$ for $m \in \mathbb{R}^{n}$ has the same properties.

Since

$$
\begin{gathered}
x(a, s)=m, \\
x(b, s)=\Omega(a, b) m+y_{0}(b, s),
\end{gathered}
$$

one can reduce the equations $L x(a, s)=l, R x(b, s)=r$ to system

$$
\left(\begin{array}{c}
L \\
R \Omega(a, b)
\end{array}\right) m=\left(\begin{array}{c}
l \\
r-R y_{0}(b, s)
\end{array}\right) .
$$

Since the determinant of this system is assumed to be non-zero, $m$ is determined uniquely.

We begin with the construction of the matrix $G(t, s)$. Vector-columns $g_{p}(t, s)(1 \leq p \leq n)$ satisfy the differential equations

$$
\begin{gathered}
\frac{d}{d t} g_{p}(t, s)=A(t) g_{p}(t, s), \quad a<t<s, s<t<b \\
L g_{p}(a, s)=0, \quad R g_{p}(b, s)=0,
\end{gathered}
$$




$$
g_{p}(s+0, s)-g_{p}(s-0, s)=\left(\begin{array}{c}
0 \\
\cdots \\
0 \\
1 \\
0 \\
\cdots \\
0
\end{array}\right)-p \text { position. }
$$

By Lemma 1 vector function $g_{p}(t, s)$ is defined uniquely. Hence matrix $G(t, s)$ is determined uniquely by its columns.

The derivation of matrices $G_{L}(t)$ and $G_{R}(t)$ is more easy and we omit it (see [3, p. 112]).

Once the Green matrices have been constructed we turn to the reducing of problem (3) to the integral equation. Substituting $x(t)=\stackrel{\circ}{y}(t)+z(t)$ into (3) we get

$$
\left\{\begin{array}{c}
\frac{d z}{d t}=F_{z}^{\prime}(\stackrel{\circ}{y}, t) z+f(\stackrel{\circ}{y}+z, t, \omega t)-F(\stackrel{\circ}{y}, t)-F_{z}^{\prime}(\stackrel{\circ}{y}, t) z \quad \omega \gg 1 \\
L z(a)=0 \\
R z(b)=0 .
\end{array}\right.
$$

Then the use of the Green matrix leads to integral equation (see [3, p. 115])

$$
z(t)=\int_{a}^{b} G(t, s)\left[f(\stackrel{\circ}{y}+z, s, \omega s)-F(\stackrel{\circ}{y}, s)-F_{z}^{\prime}(\stackrel{\circ}{y}, s) z\right] d s .
$$

Substituting $x(t)=\stackrel{\circ}{y}(t)+z(t)$ into (5) we get

$$
\left\{\begin{array}{c}
\frac{d z}{d t}=F_{z}^{\prime}(\stackrel{\circ}{y}, t) z+f(\stackrel{\circ}{y}+z, t, \omega t)-F(\stackrel{\circ}{y}, t)-F_{z}^{\prime}(\stackrel{\circ}{y}, t) z \quad \omega \gg 1 \\
M z(0)=0 .
\end{array}\right.
$$

Let $B_{1}$ and $B_{2}$ be the subspaces of the exponential dichotomy of $R^{n}$ induced by ODE. Then $R^{n}=B 1 \oplus B 2$. Let $P_{1}$ and $P_{2}$ be the projectors onto subspaces $B_{1}$ and $B_{2}$.

Let $z(t)$ be bounded solution of problem (8) on the semi-axis. Then (see [4])

$$
z(t)=X(t, 0) a+\int_{0}^{\infty} G_{1}(t, s)\left(f(\stackrel{\circ}{y}(s)+z(s), s, \omega s)-F(\stackrel{\circ}{y}(s), s)-F_{z}^{\prime}(\stackrel{\circ}{y}(s), s) z\right) d s,
$$

where $a \in B_{1}$,

$$
G_{1}(t, s)=\left\{\begin{array}{l}
U(t) P_{1} U^{-1}(s), t>s \\
-U(t) P_{2} U^{-1}(s), t<s
\end{array}\right.
$$


Let us put $a=S \alpha, \alpha \in \mathbb{R}^{k}, k=\operatorname{dim} B_{1}$

Substituting $z(0)$ into the boundary condition, we obtain

$$
0=[M S] \alpha+M \int_{0}^{\infty} G_{1}(0, s)\left(f(\stackrel{\circ}{y}(s)+z(s), s, \omega s)-F(\stackrel{\circ}{y}(s), s)-F_{z}^{\prime}(\stackrel{\circ}{y}(s), s) z\right) d s .
$$

Then

$$
\alpha=-[M S]^{-1} M \int_{0}^{\infty} G_{1}(0, s)\left(f(\stackrel{\circ}{y}(s)+z(s), s, \omega s)-F(\stackrel{\circ}{y}(s), s)-F_{z}^{\prime}(\stackrel{\circ}{y}(s), s) z\right) d s .
$$

Thus, the integer equation we are looking for has the form

$$
\begin{aligned}
& z(t)=-X(t, 0) S[M S]^{-1} M \int_{0}^{\infty} G_{1}(0, s)(f(\stackrel{\circ}{y}(s)+z(s), s, \omega s)-F(\stackrel{\circ}{y}(s), s)- \\
& \left.-F_{z}^{\prime}(\stackrel{\circ}{y}(s), s) z\right) d s+\int_{0}^{\infty} G_{1}(t, s)\left(f(\stackrel{\circ}{y}(s)+z(s), s, \omega s)-F(\stackrel{\circ}{y}(s), s)-F_{z}^{\prime}(\stackrel{\circ}{y}(s), s) z\right) d s .
\end{aligned}
$$

\section{References}

[1] V. B. Levenshtam, P. E. Shubin, Averaging of Evolution System with Boundary Conditions, Ser. A: Appl. Math. Inform. and Mech. 2013. Vol. 5, No 2. P. 61-67.

[2] B. P. Demidovich, Lectures on the mathematical theory of stability, Nauka, Moscow, 1967.

[3] S. K. Godunov, Ordinary differential equations with constant coefficient, Publishing University of Novosibirsk, Novosibirsk, 1994.

[4] Y. L. DaletskiI, M. G. KRein, Stability of solutions of differential equations in Banach spaces, Nauka, Moscow, 1970.

[5] I. B. Simonenko, A Justification of the averaging method for abstract parabolic equations, Math. USSR Sb. $1051 ., 1970$.

[6] I. B. Simonenko, The averaging method in the theory of nonlinear equations of parabolic type with application to problems of hydrodynamic stability, PRU, Rostov-on-Don, 1989. 